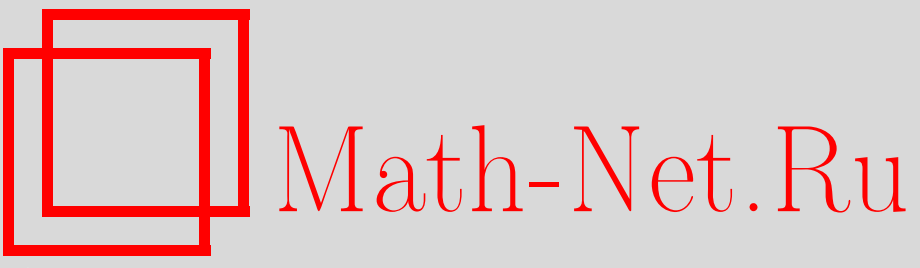

E. S. Zhukovskiy, W. Merchela, On the continuous dependence on the parameter of the set of solutions of the operator equation, Izv. IMI UdGU, 2019, Volume 54, 27-37

DOI: https://doi.org/10.20537/2226-3594-2019-54-02

Use of the all-Russian mathematical portal Math-Net.Ru implies that you have read and agreed to these terms of use

http: //www.mathnet.ru/eng/agreement

Download details:

IP : 18.234 .156 .22

April 26, 2023, 04:48:42 


\section{УДК 517.988}

\section{(C) Е. С. Жуковский, В. Мерчела}

\section{О НЕПРЕРЫВНОЙ ЗАВИСИМОСТИ ОТ ПАРАМЕТРА МНОЖЕСТВА РЕШЕНИЙ ОПЕРАТОРНОГО УРАВНЕНИЯ}

Для отображений, действующих из метрического пространства $\left(X, \rho_{X}\right)$ в пространство $Y$, на котором определено расстояние (то есть отображение $d: X \times X \rightarrow \mathbb{R}_{+}$такое, что $d(x, u)=0 \Leftrightarrow x=u$ ), определяется следующий аналог свойства накрывания. Множеством $\alpha$-накрывания отображения $f: X \rightarrow Y$ названо множество

$$
\operatorname{Cov}_{\alpha}[f]=\left\{(x, \tilde{y}) \in X \times Y: \exists \tilde{x} \in X f(\tilde{x})=\tilde{y}, \rho_{X}(\tilde{x}, x) \leqslant \alpha^{-1} d_{Y}(\tilde{y}, f(x))\right\} .
$$

Для заданных $\tilde{y} \in Y, \Phi: X \times X \rightarrow Y$ рассматривается уравнение $\Phi(x, x)=\tilde{y}$. Сформулирована теорема о существовании решения. Исследуется проблема устойчивости решений к малым изменениям отображения $\Phi$. А именно, рассмотрена последовательность таких отображений $\Phi_{n}: X \times X \rightarrow Y, n=1,2, \ldots$, что для всех $x \in X$ выполнено $(x, \tilde{y}) \in \operatorname{Cov}_{\alpha}\left[\Phi_{n}(\cdot, x)\right]$, отображение $\Phi_{n}(x, \cdot)$ является $\beta$-липшицевым и для решения $x^{*}$ исходного уравнения имеет место сходимость $d_{Y}\left(\tilde{y}, \Phi_{n}\left(x^{*}, x^{*}\right)\right) \rightarrow 0$. При выполнении этих условий утверждается, что при любом $n$ существует $x_{n}^{*}$ такой, что $\Phi_{n}\left(x_{n}^{*}, x_{n}^{*}\right)=\tilde{y}$ и $\left\{x_{n}^{*}\right\}$ сходится к $x^{*}$ в метрическом пространстве $X$. Также в статье рассмотрено уравнение $\Phi(x, x, t)=\tilde{y}$ с параметром $t$ - элементом топологического пространства. Предполагается, что $(x, \tilde{y}) \in \operatorname{Cov}_{\alpha}\left[\Phi_{n}(\cdot, x, t)\right]$, отображение $\Phi_{n}(x, \cdot, t)$ является $\beta$-липшицевым, а отображение $\Phi_{n}(x, x, \cdot)$ - непрерывным. Доказаны утверждения о полунепрерывной сверху и снизу зависимости множества решений от параметра $t$.

Ключевые слова: операторное уравнение, существование решения, оценка решения, непрерывная зависимость решения от параметров, метрическое пространство, накрывающее отображение, липшицево отображение.

DOI: $10.20537 / 2226-3594-2019-54-02$

\section{Введение}

Теории накрывающих отображений, ее применениям в различных задачах посвящены работы Е.Р. Авакова, А. В. Арутюнова, Б. Д. Гельмана, А. В. Дмитрука, А. Д. Иоффе, А. А. Милютина, Б. С. Мордуховича, Н. П. Осмоловского, В. М. Тихомирова, А. Удерзо и других авторов. Исследования свойства накрывания начато в работах Л.А. Люстерника, Л. М. Грейвса для отображений банаховых пространств (см. [1,2]). Накрывающие отображения, действующие из метрического пространства в линейное метрическое пространство, рассмотрены в работе [3], в которой, в частности, приведена теорема Милютина об устойчивости свойства накрывания к липшицевым возмущениям. В этой теореме утверждается, что разность $\alpha$-накрывающего и $\beta$-липшицева отображений в случае $\alpha>\beta$ является $(\alpha-\beta)$ накрывающим отображением. Содержательным распространением теоремы Милютина стала теорема Арутюнова (см. [4]) о точке совпадения двух отображений - накрывающего и липшицева, действующих из одного метрического пространства в другое. В работе [5] получено утверждение о нелинейных липшицевых возмущениях накрывающих отображений, действующих в метрических пространствах, этот результат применен к исследованию разрешимости задачи Коши для неявного дифференциального уравнения. Уточнения теоремы о возмущениях, ее распространения на более широкий класс отображений получены 
в $[6,7]$. Также в этих работах получены условия устойчивости решений операторных уравнений с накрывающими отображениями в метрических пространствах к малым изменениям этих отображений. Используется подход, предложенный в $[8,9]$ для исследования задачи об устойчивости точки совпадения накрывающего и липшицева отображений. На основании утверждений об устойчивости операторных уравнений в $[6,7]$ получены условия непрерывной зависимости от параметров решений неявных дифференциальных и интегральных уравнений.

В связи с исследованиями систем уравнений, краевых задач и задач управления, кратных неподвижных точек и кратных точек совпадения, некоторых других теоретических и прикладных задач возникла потребность в распространении результатов о накрывающих отображениях на пространства с «ослабленными метриками». В $[10,11]$ определен аналог свойства накрывания для отображений, действующих в пространствах с векторнозначными метриками, и для таких отображений получены утверждения о липшицевых возмущениях. В [12] доказана теорема о точках совпадения в $\left(q_{1}, q_{2}\right)$-квазиметрических пространствах. В [13] получена теорема о точках совпадения для отображений, действующих из метрического пространства $X$ во множество $Y$, не являющееся метрическим пространством, с расстоянием $d_{Y}: Y^{2} \rightarrow \mathbb{R}_{+}$, удовлетворяющим лишь аксиоме тождества.

Настоящая работа продолжает исследование отображений, действующих из метрического пространства $X$ во множество $Y$ с расстоянием, которое не обязано быть симметричным и может не удовлетворять неравенству треугольника. В $\S 1$ определены аналоги понятий накрывания и липшицевости отображений, действующих из $X$ в $Y$. В $\S 2$ исследуется уравнение $\Phi(x, x)=\tilde{y}$ относительно неизвестного элемента $x \in X$, с заданной правой частью $\tilde{y} \in Y$ и отображением $\Phi: X^{2} \rightarrow Y$, являющимся по первому аргументу накрывающим, а по второму аргументу - липшицевым. Рассматривается задача о существовании и оценках решений, устойчивости решений к малым изменениям отображения $\Phi$ и элемента $\tilde{y}$. Так как в полученных утверждениях требования к расстоянию в $Y$ минимальны, естественное в подобных утверждениях предположение о сходимости в $Y$ образов соответствующих отображений оказывается гораздо менее обременительным, чем в цитируемых выше работах [6-9]. Таким образом, можно ожидать, что предлагаемые утверждения позволят расширить приложения теорем о накрывающих отображениях. В 33 рассмотрено уравнение $\Phi(x, x, t)=\tilde{y}$, содержащее параметр $t$ - элемент некоторого топологического пространства. Получены условия полунепрерывной сверху и снизу зависимости множества решений этого уравнения от параметра $t$.

\section{$\S 1$. Основные понятия}

Пусть $X=\left(X, \rho_{X}\right)$ - метрическое пространство. Обозначим замкнутый шар в $X$ с центром в точке $x_{0} \in X$ радиуса $r$ через $B_{X}\left(x_{0}, r\right)=\left\{x \in X: \rho_{X}\left(x, x_{0}\right) \leqslant r\right\}$.

Пусть задано непустое множество $Y$, на котором определена функция расстояния отображение $d_{Y}: Y^{2} \rightarrow \mathbb{R}_{+}$, удовлетворяющее аксиоме тождества

$$
\forall y_{1}, y_{2} \in Y \quad d_{Y}\left(y_{1}, y_{2}\right)=0 \quad \Leftrightarrow \quad y_{1}=y_{2}
$$

Под сходимостью при $i \rightarrow \infty$ последовательности $\left\{y_{i}\right\} \subset Y$ к элементу $y \in Y$ будем понимать сходимость $d_{Y}\left(y, y_{i}\right) \rightarrow 0$ (вследствие невыполнения неравенства треугольника предел $y$ может быть не единственным, а вследствие несимметричности расстояния в $Y$ такая сходимость не равносильна сходимости $\left.d_{Y}\left(y_{i}, y\right) \rightarrow 0\right)$.

Для отображений, действующих из метрического пространства $X$ во множество $Y$ с расстоянием $d_{Y}$, стандартно определяются понятия непрерывности и замкнутости (см. [13]). Отображение $f: X \rightarrow Y$ является непрерывным в точке $x \in X$, если для любой сходящейся к $x$ последовательности $\left\{x_{i}\right\} \subset X$ выполнено $d_{Y}\left(f(x), f\left(x_{i}\right)\right) \rightarrow 0$. Отображение 
$f: X \rightarrow Y$ является замкнутым в точке $x \in X$, если для любой сходящейся к $x$ последовательности $\left\{x_{i}\right\} \subset X$ из существования $y \in Y$ такого, что $d_{Y}\left(y, f\left(x_{i}\right)\right) \rightarrow 0$, следует равенство $f(x)=y$. Отображение, непрерывное в каждой точке (замкнутое в каждой точке), называем непрерывным (замкнутым). Отображение $f: X \rightarrow Y$ называем замкнутым относительно шара $B_{X}\left(x_{0}, r\right)$ и одноточечного множества $\{y\} \subset Y$, если для любой последовательности $\left\{x_{i}\right\} \subset B_{X}\left(x_{0}, r\right)$ и любого $x \in X$ из сходимостей $x_{i} \rightarrow x$ и $d_{Y}\left(y, f\left(x_{i}\right)\right) \rightarrow 0$, следует, что $f(x)=y$ (в частном случае при $r=\infty$ получаем определение замкнутости относительно всего $X$ и множества $\{y\}$ ). Приведенное определение аналогично определению [14, Definition 3] замкнутости отображения относительно множеств метрических пространств.

О п р е д е л е н и е 1 . Пусть $\alpha>0$. Будем называть множеством $\alpha$-накрывания отображения $f: X \rightarrow Y$ множество всех пар $(x, \tilde{y}) \in X \times Y$, для каждой из которых существует $\tilde{x} \in X$ такой, что

$$
f(\tilde{x})=\tilde{y} \quad \text { и } \quad \rho_{X}(\tilde{x}, x) \leqslant \frac{1}{\alpha} d_{Y}(\tilde{y}, f(x)) .
$$

Множество $\alpha$-накрывания отображения $f$ будем обозначать символом $\operatorname{Cov}_{\alpha}[f]$.

Если $\operatorname{Cov}_{\alpha}[f]=X \times Y$, то отображение $f$, естественно, называем $\alpha$-накрывающим (соответствующее определение для отображений метрических пространств см. [4]).

О п р е д е л е н и е 2. Пусть $\beta \geqslant 0$. Будем называть множеством $\beta$-липшицевости отображения $f: X \rightarrow Y$ множество всех пар $(x, \tilde{y}) \in X \times Y$, удовлетворяющих соотношению

$$
\forall \tilde{x} \in X \quad f(\tilde{x})=\tilde{y} \quad \Rightarrow \quad d_{Y}(\tilde{y}, f(x)) \leqslant \beta \rho_{X}(\tilde{x}, x) .
$$

Множество $\beta$-липшицевости отображения $f$ будем обозначать символом $\operatorname{Lip}_{\beta}[f]$.

Если $\operatorname{Lip}_{\beta}[f]=X \times Y$, то отображение $f$ называем $\beta$-липшицевым. В этом случае для любых $x_{1}, x_{2} \in X$ справедливо неравенство

$$
d_{Y}\left(f\left(x_{1}\right), f\left(x_{2}\right)\right) \leqslant \beta \rho_{X}\left(x_{1}, x_{2}\right) .
$$

Как и для $\beta$-липшицевых отображений «обычных» метрических пространств, $\beta$-липшицево отображение в рассматриваемых здесь пространствах является непрерывным. В случае если $\operatorname{Lip}_{\beta}[f] \neq X \times Y$, то, вообще говоря, непрерывность $f$ в каких либо точках пространства $X$ гарантировать нельзя.

\section{§2. Операторное уравнение}

Пусть задано отображение $\Phi: X^{2} \rightarrow Y$ и элемент $\tilde{y} \in Y$. Определим отображение

$$
F: X \rightarrow Y, F(x)=\Phi(x, x),
$$

и рассмотрим уравнение

$$
F(x)=\tilde{y} .
$$

Следующее утверждение о разрешимости уравнения (2.2) получено в [15].

Т е о р е м а 1. Пусть метрическое пространство $X$ является полным, $x_{0} \in X, 0 \leqslant \beta<$ $\alpha$. Положим

$$
r=\frac{1}{\alpha-\beta} d_{Y}\left(\tilde{y}, F\left(x_{0}\right)\right) .
$$

Пусть при любом $x \in B_{X}\left(x_{0}, r\right)$ пара $(x, \tilde{y})$ принадлежит множеству $\operatorname{Cov}_{\alpha}[\Phi(\cdot, x)] \alpha$ накрывания отображения $\Phi(\cdot, x): X \rightarrow Y$, а также множеству $\operatorname{Lip}_{\beta}[\Phi(x, \cdot)] \beta$-липшицевости отображения $\Phi(x, \cdot): X \rightarrow Y$, и отображение $F: X \rightarrow Y$ является замкнутым относительно множеств $B_{X}\left(x_{0}, r\right) u\{\tilde{y}\}$. Тогда уравнение (2.2) имеет решение в шаре $B_{X}\left(x_{0}, r\right)$. 
Основным отличием теоремы 1 от теорем о разрешимости уравнения (2.2), полученных в [5-7], являются существенно меньшие требования к пространству $Y$. В теореме 1 расстояние $d_{Y}$ не является метрикой, а только удовлетворяет аксиоме тождества (1.1). Отметим также, что используемые в теореме 1 условия принадлежности пары $(x, \tilde{y})$ при любом $x \in B_{X}\left(x_{0}, r\right)$ множествам $\operatorname{Cov}_{\alpha}[\Phi(\cdot, x)]$ и $\operatorname{Lip}_{\beta}[\Phi(x, \cdot)]$ ослабляют используемые в аналогичных утверждениях предположения $\alpha$-накрывания отображения $\Phi(\cdot, x)$ и $\beta$-липшицевости отображения $\Phi(x, \cdot)$. В теореме 1 также уточнено предположение замкнутости отображения $F$.

Отметим, что цитируемая выше статья [15], в которой получена теорема 1, не содержит примеры, подтверждающие, что ее предположения менее ограничительны, чем используемые в [5-7]. Поэтому приведем здесь пример отображения, удовлетворяющего условиям теоремы 1, действующего во множество, не являющееся метрическим пространством. Это обстоятельство не позволяет применить результаты [5-7], в то же время, теорема 1 гарантирует существование решения соответствующую операторного уравнения и его оценку.

$\Pi$ р и м е $\mathrm{p} 1$. Обозначим через $\mathbb{N}, \mathbb{Z}$ множества натуральных и целых чисел, соответственно, $\mathbb{Z}_{+}=\mathbb{N} \cup\{0\}$; символом [ $\left.\cdot\right]$ обозначим целую часть действительного числа. На множестве $X=\left\{x_{i}, i \in \mathbb{Z}\right\}$ определим расстояние $\rho_{X}: X^{2} \rightarrow \mathbb{R}_{+}$соотношениями:

$$
\begin{gathered}
\rho_{X}\left(x_{i}, x_{i}\right)=0, \quad i \in \mathbb{Z} ; \\
\rho_{X}\left(x_{i}, x_{i+1}\right)=\rho_{X}\left(x_{i+1}, x_{i}\right)= \begin{cases}1, & i=2 k, \quad k \in \mathbb{Z}_{+}, \\
\frac{1}{k+2}, & i=2 k+1, \quad k \in \mathbb{Z}_{+} ;\end{cases} \\
\rho_{X}\left(x_{-i}, x_{-i-1}\right)=\rho_{X}\left(x_{-i-1}, x_{-i}\right)=\frac{1}{2\left(\left[\frac{i}{2}\right]+2\right)}, \quad i \in \mathbb{Z}_{+} ; \\
\rho_{X}\left(x_{i}, x_{i+m}\right)=\rho_{X}\left(x_{i+m}, x_{i}\right)=\sum_{j=i}^{i+m-1} \rho_{X}\left(x_{j}, x_{j+1}\right), \quad i \in \mathbb{Z}, m \in \mathbb{N} .
\end{gathered}
$$

Очевидно, эта функция является метрикой.

Покажем, что метрическое пространство $X$ является полным. Любая последовательность, содержащая бесконечно много различных элементов этого множества, не является фундаментальной, так как для любого $i$ вследствие расходимости гармонического ряда выполнено $\lim _{j \rightarrow \infty} \rho_{X}\left(x_{i}, x_{j}\right)=\infty, \lim _{j \rightarrow-\infty} \rho_{X}\left(x_{i}, x_{j}\right)=\infty$. Таким образом, фундаментальной может быть только последовательность, которая начиная с некоторого номера постоянна, и такая последовательность, очевидно, сходится.

Далее, на множестве $Y=\left\{y_{i}, i \in \mathbb{Z}\right\}$ зададим расстояние $d_{Y}: Y^{2} \rightarrow \mathbb{R}_{+}$следующими соотношениями. Для любого $i \in \mathbb{Z}_{+}$будем полагать

$$
\begin{aligned}
& d_{Y}\left(y_{i}, y_{i+m}\right)=d_{Y}\left(y_{i+m}, y_{i}\right)= \begin{cases}k, & m=2 k, k \in \mathbb{Z}_{+}, \\
k+\frac{1}{\left[\frac{i+2 k}{2}\right]+2}, & m=2 k+1, k \in \mathbb{Z}_{+}\end{cases} \\
& d_{Y}\left(y_{-i}, y_{-i}\right)=0 ; \quad d_{Y}=\left(y_{-i}, y_{-i-1}\right)=d_{Y}\left(y_{-i-1}, y_{-i}\right)=\left\{\begin{array}{l}
2, i=2 k, k \in \mathbb{Z}_{+} \\
1, i=2 k+1, k \in \mathbb{Z}_{+}
\end{array}\right. \\
& d_{Y}\left(y_{-i}, y_{-i-m}\right)=d_{Y}\left(y_{-i-m}, y_{-i}\right)=\sum_{s=i}^{i+m-1} d_{Y}\left(y_{-s}, y_{-s-1}\right), \quad m \in \mathbb{N} ; \\
& d_{Y}\left(y_{-i}, y_{m}\right)=d_{Y}\left(y_{m}, y_{-i}\right)=d_{Y}\left(y_{-i}, y_{0}\right)+d_{Y}\left(y_{0}, y_{m}\right), \quad m \in \mathbb{N} .
\end{aligned}
$$

Определенное этими соотношениями расстояние $d_{Y}$ симметрично, что позволяет вместо обозначения $d_{Y}\left(y, y_{i_{n}}\right) \rightarrow 0$ сходимости последовательности $\left\{y_{i_{n}}\right\} \subset Y$ при $n \rightarrow \infty$ к 
элементу $y \in Y$ пользоваться обозначением $y_{i_{n}} \rightarrow y$. Очевидно, что пространство $\left(Y, d_{Y}\right)$ не является метрическим, так как при любом $i \in \mathbb{N}$ выполнено $d_{Y}\left(y_{i}, y_{i+1}\right)+d_{Y}\left(y_{i+1}, y_{i+2}\right)<$ $d_{Y}\left(y_{i}, y_{i+2}\right)$. Более того, $\left(Y, d_{Y}\right)$ не является даже $f$-квазиметрическим пространством (подробнее определение и свойства $f$-квазиметрических пространств см. [12]). Действительно, для последовательностей $\left\{y_{i}\right\},\left\{y_{i+1}\right\},\left\{y_{i+2}\right\}$ имеют место сходимости $d_{Y}\left(y_{i}, y_{i+1}\right) \rightarrow 0$, $d_{Y}\left(y_{i+1}, y_{i+2}\right) \rightarrow 0$, однако, $d_{Y}\left(y_{i}, y_{i+2}\right)=1$.

Определим отображение $\Phi: X^{2} \rightarrow Y$ соотношением

$$
\Phi\left(x_{i}, x_{j}\right)=y_{-i}, \quad \forall i, j \in \mathbb{Z}
$$

и проверим, удовлетворяет ли оно условиям теоремы 1.

Отображение $\Phi\left(x_{i}, \cdot\right)$ постоянно, т. е. является липшицевым с коэффициентом $\beta=0$. Отображение $\Phi\left(\cdot, x_{j}\right)$ (как и любое определенное на данном пространстве $X$ отображение) является непрерывным, поскольку для любой сходящейся к некоторому элементу $u \in X$ последовательности $\left\{x_{i_{n}}\right\} \subset X$ существует такое $n_{0}$, что при всех $n \geqslant n_{0}$ выполнено $x_{i_{n}}=$ $u$. Тогда $\Phi\left(x_{i_{n}}, x_{j}\right)=\Phi\left(u, x_{j}\right)$ и, таким образом, $\Phi\left(x_{i_{n}}, x_{j}\right) \rightarrow \Phi\left(u, x_{j}\right)$. При любом $x \in X$ отображение $\Phi(\cdot, x): X \rightarrow Y$ является сюръективным и накрывающим с коэффициентом

$$
\alpha=\min _{i, j, l \in \mathbb{Z}}\left\{\frac{d_{Y}\left(\Phi\left(x_{i}, x_{j}\right), \Phi\left(x_{l}, x_{j}\right)\right)}{\rho_{X}\left(x_{i}, x_{l}\right)}\right\}=\min _{i, j \in \mathbb{Z}}\left\{\frac{d_{Y}\left(y_{-i}, y_{-l}\right)}{\rho_{X}\left(x_{i}, x_{l}\right)}\right\}=2>\beta=0 .
$$

Итак, для любых $\tilde{y} \in Y, x_{0} \in X$ выполнены все условия теоремы 1 , и операторное уравнение $F(x) \equiv \Phi(x, x)=\tilde{y}$ имеет решение в шаре $B_{X}\left(x_{0}, r\right)$, радиус которого определяется формулой (2.3). Результаты [5-7] в данном случае не применимы.

Используя теорему 1 , получим условия устойчивости решений уравнения (2.2) к малым изменениям отображения $F$.

Пусть при каждом $n \in \mathbb{N}$ заданы отображение $\Phi_{n}: X^{2} \rightarrow Y$ и элемент $\tilde{y}_{n} \in Y$. Определим отображение $F_{n}: X \rightarrow Y, F_{n}(x)=\Phi_{n}(x, x)$ и рассмотрим уравнение

$$
F_{n}(x)=\tilde{y}_{n} .
$$

Нас будет интересовать вопрос о сходимости решений уравнения (2.4) при $n \rightarrow \infty$ к решению уравнения (2.2) в случае, если есть некоторая сходимость $F_{n}$ к $F$ и $\tilde{y}_{n}$ к $\tilde{y}$. Ответ на этот вопрос дает следующее утверждение.

Т е о р е м а 2. Пусть метрическое пространство $X$ является полным, известно решение $x^{*}$ уравнения (2.2), при каждом $n \in \mathbb{N}$ задань числа $0 \leqslant \beta_{n}<\alpha_{n}$. Положим

$$
r_{n}=\frac{1}{\alpha_{n}-\beta_{n}} d_{Y}\left(\tilde{y}_{n}, F_{n}\left(x^{*}\right)\right) .
$$

Пусть для каждого $n \in \mathbb{N}$ при любом $x \in B_{X}\left(x^{*}, r_{n}\right)$ пара $\left(x, \tilde{y}_{n}\right)$ принадлежит множеству $\operatorname{Cov}_{\alpha_{n}}\left[\Phi_{n}(\cdot, x)\right] \alpha_{n}$-накрывания отображения $\Phi_{n}(\cdot, x): X \rightarrow Y$, а также множеству $\operatorname{Lip}_{\beta_{n}}\left[\Phi_{n}(x, \cdot)\right] \beta_{n}$-липшицевости отображения $\Phi_{n}(x, \cdot): X \rightarrow Y$ и отображение $F_{n}: X \rightarrow Y$ является замкнутым относительно множеств $B_{X}\left(x^{*}, r_{n}\right) u\left\{\tilde{y}_{n}\right\}$. Если при $n \rightarrow \infty$ выполнено $r_{n} \rightarrow 0$, то при любом $n \in \mathbb{N}$ уравнение (2.4) разрешимо и существует такое его решение $x_{n}^{*}$, что при $n \rightarrow \infty$ имеет место сходимость $x_{n}^{*} \rightarrow x^{*}$ в $X$.

Д о к а з а т е л ь с в о. При любом $n$ для уравнения (2.4) выполнены условия теоремы 1 , где $x_{0}=x^{*}$. Следовательно, при любом $n$ существует решение $x_{n}^{*}$ уравнения (2.4) такое, что $\rho_{X}\left(x_{n}^{*}, x^{*}\right) \leqslant r_{n}$. Из этого неравенства, в силу сходимости $r_{n} \rightarrow 0$, получаем $x_{n}^{*} \rightarrow x^{*}$ при $n \rightarrow \infty$.

Отметим, что условие $r_{n} \rightarrow 0$ выполнено, например, в случае, когда имеет место сходимость $d_{Y}\left(\tilde{y}_{n}, F_{n}\left(x^{*}\right)\right) \rightarrow 0$, а константы накрывания и липшицевости отображений $\Phi_{n}(\cdot, x), \Phi_{n}(x, \cdot)$ не зависят от $n$. 


\section{§3. Непрерывная зависимость решений от параметров}

Теорему 1 можно применить к задаче о непрерывной зависимости от параметров решений уравнений.

Введем необходимые обозначения и напомним определения полунепрерывности сверху и снизу многозначных отображений (подробнее см. [16, § 2.3], [17, § 1.2]).

Пусть $M$ - подмножество метрического пространства $X, r>0$. Обозначим $r$-раздутие $\left\{x \in X: \exists x_{0} \in M \rho_{X}\left(x, x_{0}\right)<r\right\}$ множества $M$ через $O(M, r)$. Пусть задано топологическое пространство $T$. Для $t_{0} \in T$ обозначим $\mathcal{T}\left(t_{0}\right)-$ совокупность окрестностей точки $t_{0}$. Отображение $G$, которое каждому $t \in T$ сопоставляет непустое замкнутое множество $G(t) \subset X$ называют многозначным отображением. Для такого многозначного отображения будем использовать обозначение $G: T \rightrightarrows X$. Многозначное отображение $G$ называют полунепрерывным снизу в точке $t_{0} \in T$, если для любого открытого множества $V \subset X$ такого, что $G\left(t_{0}\right) \cap V \neq \emptyset$, существует окрестность $U\left(t_{0}\right)$ точки $t_{0}$, для которой $G(t) \cap V \neq \emptyset$ при всех $t \in U\left(t_{0}\right)$. Многозначное отображение $G$ является полунепрерывным снизу в точке $t_{0} \in T$ тогда и только тогда, когда справедливо соотношение

$$
\forall x_{0} \in G\left(t_{0}\right) \quad \forall \varepsilon>0 \quad \exists U\left(t_{0}\right) \in \mathcal{T}\left(t_{0}\right) \quad \forall t \in U\left(t_{0}\right) \quad \exists x \in G(t) \quad \rho_{X}\left(x, x_{0}\right)<\varepsilon .
$$

Многозначное отображение $G$ называют полунепрерывным сверху в точке $t_{0} \in T$, если для любого открытого множества $V \subset X$ такого, что $G\left(t_{0}\right) \subset V$, существует окрестность $U\left(t_{0}\right)$ точки $t_{0}$, для которой $G\left(U\left(t_{0}\right)\right) \subset V$. Если отображение $G$ полунепрерывно сверху и снизу в точке $t_{0}$, то говорят, что оно непрерывно в этой точке. Многозначное отображение называется полунепрерывным снизу (полунепрерывным сверху, непрерывным), если оно полунепрерывно снизу (полунепрерывно сверху, непрерывно) в каждой точке $t_{0} \in T$.

Наряду с приведенными «топологическими» определениями нам потребуются определения полунепрерывности и непрерывности многозначного отображения, связанные с метрикой Хаусдорфа. Отображение $G$ называют $h$-полунепрерывным снизу ( $h$-полунепрерывным сверху) в точке $t_{0} \in T$, если для любого $\varepsilon>0$ существует окрестность $U\left(t_{0}\right)$ точки $t_{0}$ такая, что при любом $t \in U\left(t_{0}\right)$ справедливо вложение $G\left(t_{0}\right) \subset O(G(t), \varepsilon)$ (вложение $G(t) \subset O\left(G\left(t_{0}\right), \varepsilon\right)$, соответственно). Отметим, что из $h$-полунепрерывности снизу отображения следует его полунепрерывность снизу, а полунепрерывное сверху отображение является $h$-полунепрерывным сверху (см. [17, п. 1.2.3]). Если отображение $G h$-полунепрерывно сверху и снизу в точке $t_{0}$, то оно называется $h$-непрерывным в этой точке. Многозначное отображение называется $h$-полунепрерывным снизу ( $h$-полунепрерывным сверху, $h$-непрерывным), если оно $h$-полунепрерывно снизу ( $h$-полунепрерывно сверху, $h$ непрерывно) в каждой точке $t_{0} \in T$.

Теперь рассмотрим задачу о непрерывной зависимости от параметров решений уравнений. Пусть задано отображение $\Phi: X \times X \times T \rightarrow Y$. Определим отображение $F: X \times T \rightarrow Y$ соотношением $F(x, t)=\Phi(x, x, t)$. Будем говорить, что отображение $F(x, \cdot): T \rightarrow Y$ равностепенно непрерывно в точке $t_{0} \in T$, если для любого $\varepsilon>0$ существует окрестность $U\left(t_{0}\right)$ точки $t_{0}$ такая, что $d_{Y}\left(F\left(x, t_{0}\right), F(x, t)\right)<\varepsilon$ при любом $t \in U\left(t_{0}\right)$ и любом $x \in X$.

Рассмотрим уравнение

$$
F(x, t)=\tilde{y}
$$

с параметром $t \in T$ относительно неизвестного $x \in X$. Обозначим множество решений этого уравнения через $\mathcal{R}(t)$.

Т е о р е м а 3. Пусть метрическое пространство $X$ является полным, $t_{0} \in T, 0 \leqslant$ $\beta<\alpha$. Пусть существует такая окрестность $V\left(t_{0}\right)$ точки $t_{0}$, что при любых $x \in X$, $t \in V\left(t_{0}\right)$ пара $(x, \tilde{y})$ принадлежит множеству $\operatorname{Cov}_{\alpha}[\Phi(\cdot, x, t)] \alpha$-накрывания отображения $\Phi(\cdot, x, t): X \rightarrow Y$ а также множеству $\operatorname{Lip}_{\beta}[\Phi(x, \cdot, t)] \beta$-липшицевости отображения 
$\Phi(x, \cdot, t): X \rightarrow Y$ и отображение $F(\cdot, t): X \rightarrow Y$ является замкнутым относительно всего $X$ и одноточечного множества $\{\tilde{y}\}$. Тогда при любом $t \in V\left(t_{0}\right)$ множество $\mathcal{R}(t)$ решений уравнения (3.7) не пусто и замкнуто в $X$. Если, кроме того, при любом $x \in X$ отображение $F(x, \cdot): T \rightarrow Y$ непрерывно в точке $t_{0}$, то многозначное отображение $\mathcal{R}: V\left(t_{0}\right) \rightrightarrows X$ является полунепрерывным снизу в точке $t_{0}$, а если отображение $F(x, \cdot): T \rightarrow Y$ равностепенно непрерывно в точке $t_{0}$, то многозначное отображение $\mathcal{R}: V\left(t_{0}\right) \rightrightarrows X$ является h-непрерывным в точке $t_{0}$.

Д о к а з а т е л ь с т в о. Из теоремы 1 следует, что при любом $t \in V\left(t_{0}\right)$ множество $\mathcal{R}(t)$ не пусто. Докажем замкнутость этого множества. Пусть последовательность $\left\{x_{i}^{*}\right\} \subset \mathcal{R}(t)$ сходится к элементу $x^{*} \in X$. Так как $F\left(x_{i}^{*}, t\right)=\tilde{y}, i=1,2, \ldots$, в силу предположения замкнутости отображения $F(\cdot, t): X \rightarrow Y$ относительно $X$ и $\{\tilde{y}\}$, имеем $F\left(x^{*}, t\right)=\tilde{y}$. Таким образом, $x^{*} \in \mathcal{R}(t)$. Итак, множество $\mathcal{R}(t)$, действительно, является замкнутым.

Покажем, что в случае непрерывности отображения $F\left(x_{0}, \cdot\right): T \rightarrow Y$ в точке $t_{0}$ отображение $\mathcal{R}: V\left(t_{0}\right) \rightrightarrows X$ полунепрерывно снизу в той же точке $t_{0}$. Проверим для $\mathcal{R}$ справедливость соотношения (3.6). Пусть $x_{0} \in \mathcal{R}\left(t_{0}\right)$, т. е. $F\left(x_{0}, t_{0}\right)=\tilde{y}$. Так как отображение $F\left(x_{0}, \cdot\right)$ непрерывное, для любого $\varepsilon>0$ можно определить такую окрестность $U\left(t_{0}\right)$ точки $t_{0}$, что при всех $t \in U\left(t_{0}\right)$ выполнено

$$
d_{Y}\left(\tilde{y}, F\left(x_{0}, t\right)\right)=d_{Y}\left(F\left(x_{0}, t_{0}\right), F\left(x_{0}, t\right)\right)<(\alpha-\beta) \varepsilon .
$$

Согласно теореме 1 при любом $t \in V\left(t_{0}\right) \cap U\left(t_{0}\right)$ уравнение (3.7) имеет решение $x \in \mathcal{R}(t)$, удовлетворяющее оценке

$$
\rho_{X}\left(x, x_{0}\right) \leqslant \frac{1}{\alpha-\beta} d_{Y}\left(\tilde{y}, F\left(x_{0}, t\right)\right)<\varepsilon .
$$

Полунепрерывность снизу отображения $\mathcal{R}$ доказана.

Пусть теперь отображение $F(x, \cdot): T \rightarrow Y$ равностепенно непрерывно в точке $t_{0}$. Для произвольного $\varepsilon>0$ определим такую окрестность $U\left(t_{0}\right)$ точки $t_{0}$, что при любых $t \in U\left(t_{0}\right)$ справедливо $d_{Y}\left(F\left(x, t_{0}\right), F(x, t)\right)<\varepsilon$ для всех $x \in X$. Поэтому для любого $t \in V(t) \cap U(t)$ и для любого $x^{\prime} \in \mathcal{R}\left(t_{0}\right)$ согласно теореме 1 существует $x \in \mathcal{R}(t)$ такой, что

$$
\rho_{X}\left(x^{\prime}, x\right) \leqslant \frac{1}{\alpha-\beta} d_{Y}\left(\tilde{y}, F\left(x_{0}, t\right)\right)<\varepsilon .
$$

Таким образом, $\mathcal{R}\left(t_{0}\right) \subset O_{X}(\mathcal{R}(t), \varepsilon)$, следовательно, отображение $\mathcal{R} h$-полунепрерывно снизу в точке $t_{0}$.

Также, в силу теоремы 1 , для любого $x \in \mathcal{R}(t)$ существует $x^{\prime} \in \mathcal{R}\left(t_{0}\right)$ такой, что

$$
\rho_{X}\left(x^{\prime}, x\right) \leqslant \frac{1}{\alpha-\beta} d_{Y}(\tilde{y}, F(x, t))<\varepsilon,
$$

т. е. $\mathcal{R}(t) \subset O_{X}\left(\mathcal{R}\left(t_{0}\right), \varepsilon\right)$, поэтому отображение $\mathcal{R}$ является $h$-полунепрерывным сверху в точке $t_{0}$. Итак, $h$-непрерывность в точке $t_{0}$ отображения $\mathcal{R}$ доказана.

В условиях теоремы 3 не гарантируется полунепрерывность сверху многозначного отображения $\mathcal{R}$ и, следовательно, не гарантируется его непрерывность. Приведем соответствующий пример.

П р и м е р 2. Пусть в пространствах $T=\mathbb{R}, X=\mathbb{R}^{2}, Y=\mathbb{R}$ задана «обычная» евклидова метрика, $\tilde{y}=0 \in \mathbb{R}$. Определим отображение $\Phi: \mathbb{R}^{2} \times \mathbb{R}^{2} \times \mathbb{R} \rightarrow \mathbb{R}$ соотношением $\Phi(x, u, t)=x_{1}-t$, где $x=\left(x_{1}, x_{2}\right), u=\left(u_{1}, u_{2}\right)$. Это линейное отображение, очевидно, непрерывно. При любых $t \in \mathbb{R}, x \in \mathbb{R}^{2}$ отображение $\Phi(t, x, \cdot): \mathbb{R}^{2} \rightarrow \mathbb{R}$, очевидно, является 
липшицевым с константой $\beta=0$. Покажем, что при любых $t \in \mathbb{R}, u \in \mathbb{R}^{2}$ отображение $\Phi(\cdot, u, t): \mathbb{R}^{2} \rightarrow \mathbb{R}$ является накрывающим. При любых $x=\left(x_{1}, x_{2}\right) \in \mathbb{R}^{2}, y^{\prime} \in \mathbb{R}$ положим $x^{\prime}=\left(y^{\prime}+t, x_{2}\right) \in \mathbb{R}^{2}$ (т. е. $\left.x_{1}^{\prime}=y^{\prime}+t, x_{2}^{\prime}=x_{2}\right)$. Для этого элемента выполнено

$$
\Phi\left(x^{\prime}, u, t\right)=y^{\prime} \text { и }\left|\Phi\left(x^{\prime}, u, t\right)-\Phi(x, u, t)\right|=\left|y^{\prime}-x_{1}+t\right|=\left|x_{1}^{\prime}-x_{1}\right|=\rho_{\mathbb{R}^{2}}\left(x^{\prime}, x\right) .
$$

Таким образом, отображение $\Phi(\cdot, u, t)$ является накрывающим с константой $\alpha=1$.

Положим $F(x, t)=\Phi(x, x, t)=x_{1}-t$. Отображение $F(x, \cdot): \mathbb{R}^{2} \rightarrow \mathbb{R}$ равностепенно непрерывно. Итак, при любом $t_{0} \in \mathbb{R}$ выполнены все условия теоремы 3. Уравнение (3.7), которое здесь имеет вид $x_{1}-t=0$, разрешимо при любом $t$, его решения образуют множество $R(t)=\left\{\left(x_{1}, x_{2}\right): x_{1}=t, x_{2} \in \mathbb{R}^{+}\right\}$. Многозначное отображение $\mathcal{R}: \mathbb{R} \rightrightarrows \mathbb{R}^{2}$ является $h$-непрерывным (в любой точке $t_{0}$ ), но не обладает свойством полунепрерывности сверху ни в одной точке $t_{0} \in \mathbb{R}$.

Финансирование. Работа выполнена при финансовой поддержке Российского фонда фундаментальных исследований (проекты № 17-01-00553-a, № 17-41-680975-р_а, № 17-5112064-ННИО_а) и Минобрнауки РФ (задание № 3.8515.2017/БЧ).

\section{СПИСОК ЛИТЕРАТУРЫ}

1. Люстерник Л.А. Об условных экстремумах функционалов // Математический сборник. 1934. T. 41. № 3. C. 390-401. http://mi.mathnet.ru/msb6470

2. Graves L.M. Some mapping theorems // Duke Math. J. 1950. Vol. 17. No. 2. P. 111-114. https://doi.org/10.1215/S0012-7094-50-01713-3

3. Дмитрук А.В., Милютин А.А., Осмоловский Н.П. Теорема Люстерника и теория экстремума // УМН. 1980. Т. 35. Вып. 6 (216). С. 11-46. http://mi.mathnet.ru/umn3878

4. Арутюнов А.В. Накрывающие отображения в метрических пространствах и неподвижные точки // Доклады Академии наук. 2007. Т. 416. № 2. С. 151-155.

https://elibrary.ru/item.asp?id=9533810

5. Аваков Е.Р., Арутюнов А.В., Жуковский Е.С. Накрывающие отображения и их приложения к дифференциальным уравнениям, не разрешенным относительно производной // Дифференциальные уравнения. 2009. Т. 45. № 5. С. 613-634.

6. Арутюнов А.В., Жуковский Е.С., Жуковский С.Е. О корректности дифференциальных уравнений, не разрешенных относительно производной // Дифференциальные уравнения. 2011. Т. 47. № 11. C. $1523-1537$.

7. Arutyunov A.V., Zhukovskiy E.S., Zhukovskiy S.E. Covering mappings and well-posedness of nonlinear Volterra equations // Nonlinear Analysis: Theory, Methods and Applications. 2012. Vol. 75. No. 3. P. 1026-1044. https://doi.org/10.1016/j.na.2011.03.038

8. Арутюнов А.В. Устойчивость точек совпадения и свойства накрывающих отображений // Математические заметки. 2009. Т. 86. Вып. 2. С. 163-169. https://doi.org/10.4213/mzm8471

9. Arutyunov A.V., Avakov E.R., Zhukovskiy S.E. Stability theorems for estimating the distance to a set of coincidence points // SIAM J. Optim. 2015. Vol. 25. No. 2. P. 807-828. https://doi.org/10.1137/140980612

10. Жуковский Е.С. О возмущениях векторно накрывающих отображений и системах уравнений в метрических пространствах // Сиб. матем. журн. 2016. Т. 57. № 2. С. 297-311. https://doi.org/10.17377/smzh.2016.57.206

11. Жуковский Е.С. О точках совпадения многозначных векторных отображений метрических пространств // Математические заметки. 2016. Т. 100. Вып. 3. С. 344-362. https://doi.org/10.4213/mzm10675

12. Арутюнов А.В., Грешнов А.В. Теория $\left(q_{1}, q_{2}\right)$-квазиметрических пространств и точки совпадения // Доклады Академии наук. 2016. Т. 469. № 5. С. 527-531.

https://doi.org/10.7868/S0869565216230031 
13. Мерчела В. К теореме Арутюнова о точках совпадения двух отображений метрических пространств // Вестник Тамбовского университета. Сер. Естественные и технические науки. 2018. T. 23. № 121. C. 65-73. https://doi.org/10.20310/1810-0198-2018-23-121-65-73

14. Arutyunov A., Avakov E., Gel'man B., Dmitruk A., Obukhovskii V. Locally covering maps in metric spaces and coincidence points // Journal of Fixed Point Theory and Applications. 2009. Vol. 5. Issue 1. P. 105-127. https://doi.org/10.1007/s11784-008-0096-z

15. Бенараб С., Жуковский Е.С., Мерчела В. Теоремы о возмущениях накрывающих отображений в пространствах с расстоянием и в пространствах с бинарным отношением // Труды Института математики и механики УрО РАН. 2019. Т. 25. № 4. С. 52-63. https://doi.org/10.21538/0134-4889-2019-25-4-52-63

16. Арутюнов А.В. Лекции по выпуклому и многозначному анализу. М.: Физматлит, 2014.

17. Борисович Ю.Г., Гельман Б.Д., Мышкис А.Д., Обуховский В.В. Введение в теорию многозначных отображений и дифференциальних включений. М.: Либроком, 2011.

Поступила в редакцию 29.10.2019

Жуковский Евгений Семенович, д. ф.-м. н., профессор, директор научно-исследовательского института математики, физики и информатики, Тамбовский государственный университет им. Г.Р. Державина, 392000, Россия, г. Тамбов, ул. Интернациональная, 33.

E-mail: zukovskys@mail.ru

Мерчела Вассим, аспирант, кафедра функционального анализа, Тамбовский государственный университет им. Г. Р. Державина, 392000, Россия, г. Тамбов, ул. Интернациональная, 33;

Лаборатория прикладной математики и моделирования, Университет им. 8 Мая 1945 г., Алжир, г. Гельма.

E-mail: merchela.wassim@gmail.com

Цитирование: Е. С. Жуковский, В. Мерчела. О непрерывной зависимости от параметра множества решений операторного уравнения // Известия Института математики и информатики Удмуртского государственного университета. 2019. Т. 54. С. 27-37. 


\section{E.S. Zhukovskiy, W. Merchela}

On the continuous dependence on the parameter of the set of solutions of the operator equation

Keywords: operator equation, existence of solutions, estimation of solutions, continuous dependence of a solution on parameters, metric space, covering mapping, Lipschitz mapping.

MSC2010: 47J05, 54E40

DOI: $10.20537 / 2226-3594-2019-54-02$

For mappings acting from a metric space $\left(X, \rho_{X}\right)$ to a space $Y$, on which a distance is defined (i.e., a function $d: X \times X \rightarrow \mathbb{R}_{+}$such that $d(x, u)=0 \Leftrightarrow x=u$ ), the following analogue of the covering property is defined. The set

$$
\operatorname{Cov}_{\alpha}[f]=\left\{(x, \tilde{y}) \in X \times Y: \exists \tilde{x} \in X f(\tilde{x})=\tilde{y}, \rho_{X}(\tilde{x}, x) \leqslant \alpha^{-1} d_{Y}(\tilde{y}, f(x))\right\}
$$

is called the set of $\alpha$-covering of the mapping $f: X \rightarrow Y$. For given $\tilde{y} \in Y, \Phi: X \times X \rightarrow Y$ the equation $\Phi(x, x)=\tilde{y}$ is considered. A theorem on the existence of a solution is formulated. The problem of the stability of solutions on small perturbations of the mapping $\Phi$ is investigated. Namely, we consider a sequence of mappings $\Phi_{n}: X \times X \rightarrow Y, n=1,2, \ldots$, such that for all $x \in X$ the following holds: $(x, \tilde{y}) \in \operatorname{Cov}_{\alpha}\left[\Phi_{n}(\cdot, x)\right]$, the mapping $\Phi_{n}(x, \cdot)$ is $\beta$-Lipschitz and for the solution $x^{*}$ of the initial equation $d_{Y}\left(\tilde{y}, \Phi_{n}\left(x^{*}, x^{*}\right)\right) \rightarrow 0$. Under these conditions, it is proved that for any $n$ there exists $x_{n}^{*}$ such that $\Phi_{n}\left(x_{n}^{*}, x_{n}^{*}\right)=\tilde{y}$ and $\left\{x_{n}^{*}\right\}$ converges to $x^{*}$ in the metric space $X$. Moreover, we consider the equation $\Phi(x, x, t)=\tilde{y}$ with the parameter $t$ which is an element of a topological space. It is assumed that $(x, \tilde{y}) \in \operatorname{Cov}_{\alpha}\left[\Phi_{n}(\cdot, x, t)\right]$, the mapping $\Phi_{n}(x, \cdot, t)$ is $\beta$-Lipschitz, and the mapping $\Phi_{n}(x, x, \cdot)$ is continuous. Statements on the upper and lower semicontinuous dependence of the solutions set on the parameter $t$ are proved.

Funding. The study was funded by the Russian Foundation for Basic Research (projects numbers 1701-00553, 17-41-680975, 17-51-12064) and Ministry of Science and Higher Education of the Russian Federation in the framework of the basic part of the state task (project no. 3.8515.2017).

\section{REFERENCES}

1. Lusternik L.A. On conditional extrema of functionals, Matematicheskii Sbornik, 1934, vol. 41, no. 3, pp. 390-401 (in Russian). http://mi.mathnet.ru/eng/msb6470

2. Graves L.M. Some mapping theorems, Duke Math. J., 1950, vol. 17, no. 2, pp. 111-114. https://doi.org/10.1215/S0012-7094-50-01713-3

3. Dmitruk A.V., Milyutin A.A., Osmolovskii N.P. Lyusternik's theorem and the theory of extrema, Russian Mathematical Surveys, 1980, vol. 35, no. 6, pp. 11-51. https://doi.org/10.1070/RM1980v035n06ABEH001973

4. Arutyunov A.V. Covering mappings in metric spaces and fixed points, Doklady Mathematics, 2007, vol. 76, no. 2, pp. 665-668. https://doi.org/10.1134/S1064562407050079

5. Avakov E.R., Arutyunov A.V., Zhukovskii E.S. Covering mappings and their applications to differential equations unsolved for the derivative, Differential Equations, 2009, vol. 45, no. 5, pp. 627-649. https://doi.org/10.1134/S0012266109050024

6. Arutyunov A.V., Zhukovskii E.S., Zhukovskii S.E. On the well-posedness of differential equations unsolved for the derivative, Differential Equations, 2011, vol. 47, no. 11, pp. 1541-1555. https://doi.org/10.1134/S0012266111110012

7. Arutyunov A.V., Zhukovskiy E.S., Zhukovskiy S.E. Covering mappings and well-posedness of nonlinear Volterra equations, Nonlinear Analysis: Theory, Methods and Applications, 2012, vol. 75, no. 3, pp. 1026-1044. https://doi.org/10.1016/j.na.2011.03.038

8. Arutyunov A.V. Stability of coincidence points and properties of covering mappings, Mathematical Notes, 2009, vol. 86, issue 1-2, pp. 153-158. https://doi.org/10.1134/S0001434609070177 
9. Arutyunov A.V., Avakov E.R., Zhukovskiy S.E. Stability theorems for estimating the distance to a set of coincidence points, SIAM J. Optim., 2015, vol. 25, no. 2, pp. 807-828. https://doi.org/10.1137/140980612

10. Zhukovskiĭ E.S. Perturbations of vectorial coverings and systems of equations in metric spaces, Siberian Mathematical Journal, 2016, vol. 57, no. 2, pp. 230-241. https:// doi.org/10.1134/S0037446616020063

11. Zhukovskiy E.S. On coincidence points of multivalued vector mappings of metric spaces, Mathematical Notes, 2016, vol. 100, issue 3-4, pp. 363-379. https://doi.org/10.1134/S0001434616090030

12. Arutyunov A.V., Greshnov A.V. Theory of $\left(q_{1}, q_{2}\right)$-quasimetric spaces and coincidence points, Doklady Mathematics, 2016, vol. 94, no. 1, pp. 434-437. https://doi.org/10.1134/S1064562416040232

13. Merchela W. About Arutyunov theorem of coincidence point for two mapping in metric spaces, Vestnik Tambovskogo Universiteta. Ser. Estestvennye i Tekhnicheskie Nauki, 2018, vol. 23, no. 121, pp. 65-73. https://doi.org/10.20310/1810-0198-2018-23-121-65-73

14. Arutyunov A., Avakov E., Gel'man B., Dmitruk A., Obukhovskii V. Locally covering maps in metric spaces and coincidence points, Journal of Fixed Point Theory and Applications, 2009, vol. 5, issue 1, pp. 105-127. https://doi.org/10.1007/s11784-008-0096-z

15. Benarab S., Zhukovskii E.S., Merchela W. Trudy Instituta Matematiki i Mekhaniki URO RAN, 2019, vol. 25, no. 4, pp. 52-63 (in Russian). https://doi.org/10.21538/0134-4889-2019-25-4-52-63

16. Arutyunov A.V. Lektsii po vypuklomu i mnogoznachnomu analizu (Lectures on convex and multivalued analysis), Moscow: Fizmatlit, 2014.

17. Borisovich Yu.G., Gel'man B.D., Myshkis A.D., Obukhovskii V.V. Vvedenie v teoriyu mnogoznachnykh otobrazhenii $i$ differentsial'nykh vklyuchenii (Introduction to the theory of multivalued mappings and differential inclusions), Moscow: Librokom, 2011.

Received 29.10.2019

Zhukovskiy Evgeny Semenovich, Doctor of Physics and Mathematics, Professor, Director of the Research Institute of Mathematics, Physics and Computer Science, Derzhavin Tambov State University, ul. Internatsional'naya, 33, Tambov, 392000, Russia.

E-mail: zukovskys@mail.ru

Merchela Wassim, Post-Graduate Student, Department of Functional Analysis, Derzhavin Tambov State University, ul. Internatsional'naya, 33, Tambov, 392000, Russia;

Laboratory of Applied Mathematics and Modeling, 8 Mai 1945 Guelma University, Guelma, Algeria. E-mail: merchela.wassim@gmail.com

Citation: E. S. Zhukovskiy, W. Merchela. On the continuous dependence on the parameter of the set of solutions of the operator equation, Izvestiya Instituta Matematiki i Informatiki Udmurtskogo Gosudarstvennogo Universiteta, 2019, vol. 54, pp. 27-37. 